\title{
ASZ1 is differentially expressed in brain metastatic human breast cancer.
}

Shahan Mamoor, MS ${ }^{1}$

$3 \quad{ }^{1}$ shahanmamoor@gmail.com East Islip, NY USA

Metastasis to the brain is a clinical problem in patients with breast cancer ${ }^{1-3}$. We mined published microarray data ${ }^{4,5}$ to compare primary and metastatic tumor transcriptomes for the discovery of genes associated with brain metastasis in humans with metastatic breast cancer. We found that the ankyrin repeat, SAM and basic leucine zipper domain containing 1, encoded by ASZ1, was among the genes whose expression was most different in the brain metastases of patients with metastatic breast cancer as compared to primary tumors of the breast ${ }^{4}$. ASZ1 was also differentially expressed in the tumor cells of patients with triple negative breast cancer ${ }^{5}$. ASZ1 mRNA was present at decreased quantities in brain metastatic tissues as compared to primary tumors of the breast. Importantly, expression of ASZ1 in primary tumors was significantly correlated with patient post-progression survival. Modulation of ASZ1 expression may be relevant to the biology by which tumor cells metastasize from the breast to the brain in humans with metastatic breast cancer.

Keywords: breast cancer, metastasis, brain metastases, central nervous system metastases, the ankyrin repeat, SAM and basic leucine zipper domain containing 1, ASZ1, systems biology of breast cancer, targeted therapeutics in breast cancer. 
One report described a 34\% incidence of central nervous system metastases in patients treated with trastuzumab for breast cancer ${ }^{2}$. More recently, the NEfERT-T clinical trial ${ }^{6}$ which compared administration of either neratinib or trastuzumab in conjunction with paclitaxel demonstrated that in a randomized, controlled setting, in breast cancer patients treated with neratinib, not only was the incidence of central nervous system recurrence significantly lower, the time to central nervous system metastasis was significantly delayed as compared to patients administered trastuzumab ${ }^{6}$. The alarmingly high rate of central nervous system metastasis described, as well as data, both anecdotal ${ }^{2}$ and from a randomized, controlled setting ${ }^{6}$ illustrating that treatment with trastuzumab may be associated with these events demands an enhanced understanding of the transcriptional makeup of brain metastatic tissues to support identification of therapeutic targets, whether they are treatment related or not. We performed a global comparative analysis of primary and metastatic tumors in patients with brain metastatic breast cancer ${ }^{4,5}$. We discovered differential and increased expression of the gene encoding the ankyrin repeat, SAM and basic leucine zipper domain containing $1, \mathrm{ASZ} 1$, in brain metastatic tissues of patients with metastatic breast cancer.

\section{Methods}

We used datasets GSE10893 ${ }^{4}$ and GSE38959 for this global differential gene expression analysis of brain metastatic breast cancer in conjunction with GEO2R. GSE10893 was generated using Agilent-011521 Human 1A Microarray G4110A technology with $n=11$ primary breast tumors and $n=3$ brain metastases from patients with breast cancer; analysis was performed using platform GPL885. GSE38959 was generated using Agilent-014850 Whole Human Genome Microarray 4x44K G4112F with $n=13$ samples of normal mammary gland ductal cells and $n=30$ samples of primary tumor cells from patients with triple negative breast cancer; analysis was performed using platform GPL4133. The Benjamini and Hochberg method of $p$-value adjustment was used for ranking of differential expression but raw $p$-values were used to assess statistical significance of global differential expression. Log-transformation of data was auto-detected, and the NCBI generated category of platform annotation was used. A statistical test was performed to evaluate whether ASZ1 gene expression was significantly different between primary tumors of the breast and brain metastases in humans with breast cancer using a two-tailed t-test. For Kaplan-Meier survival analysis, we used the Kaplan-Meier plotter online tool ${ }^{7}$ for correlation of ASZ1 mRNA expression levels with post-progression survival in $n=173$ breast cancer patients.

\section{$\underline{\text { Results }}$}

We performed global comparative transcriptome analysis of metastatic tumor tissues of patients with metastatic breast cancer using published microarray data ${ }^{4,5}$ to describe the transcriptional landscape of brain metastasis in human breast cancer in an unbiased fashion and for the discovery of novel therapeutic targets.

\section{ASZ1 is differentially expressed in the brain metastases of patients with brain metastatic breast cancer.}

Through blind, systems-level analysis of published microarray data ${ }^{4}$, we identified the ankyrin repeat, SAM and basic leucine zipper domain containing 1, encoded by ASZ1, as a differentially expressed gene in the breast metastatic tissues of humans with breast cancer (Table 1). When sorting each of the genes expressed in brain metastases based on significance of difference as compared to primary tumors of the breast in patients with breast cancer, ASZ1 ranked 210 out of 17418 total transcripts (Chart 1), equating to $98.8 \%$ differential expression. Differential expression of ASZ1 in the brain metastases of patients with metastatic breast cancer was statistically significant (Chart $1 ; p=5.71 \mathrm{e}-04)$. 
To attempt to validate differential transcriptome-wide differential expression of ASZ1 in human breast cancer, we queried a second microarray datase $t^{5}$, here comparing primary tumor cells from patients with triple negative breast cancer to normal mammary gland ductal cells. Again, we identified ASZ1 as a differentially expressed gene in the tumor tissues of patients with breast cancer (Chart 2). When sorting each of the genes expressed in triple negative breast cancer tumor cells based on significance of difference as compared to normal mammary gland ductal cells, ASZ1 ranked 14159 out of 45015 total transcripts (Chart 2), equating to $68.5 \%$ differential expression. Differential expression of ASZ1 in the brain metastases of patients with metastatic breast cancer was statistically significant (Chart $2 ; p=3.53 \mathrm{e}-02$ ). Thus, differential expression of ASZ1, transcriptome-wide, in the tumor tissues of women with breast cancer was conserved across two independent microarray datasets, both when in primary and metastatic tumor tissues.

\section{ASZ1 is expressed at lower levels in the brain metastases of patients with metastatic breast cancer.}

We obtained exact mRNA expression levels for ASZ1, in primary tumors of the breast and in brain metastasis of patients with brain metastatic breast cancer to determine direction and statistical significance of change in ASZ1 expression in brain metastatic tissues. We observed significantly lower expression of ASZ1 in the brain metastases of patients with breast cancer as compared to primary tumors of the breast: ASZ1 was expressed at $-0.02 \pm 0.38$ arbitrary units (AU) in primary tumors of the breast, while it was expressed at $-1.55 \pm 1.15$ AU in brain metastatic tissues. The difference in ASZ1 mRNA levels between primary tumors of the breast and brain metastatic tissues was statistically significant (Figure 1; $p=0.001325)$.

ASZ1 expression is significantly correlated with survival outcomes in human breast cancer.

We performed Kaplan-Meier survival analysis ${ }^{8}$ in 173 breast cancer patients in total, to evaluate whether ASZ1 tumor expression was correlated with survival outcomes in human breast cancer. We observed a statistically significant correlation between primary tumor expression of ASZ1 and post-progression survival (PPS) in patients with breast cancer (Figure 2). Patients whose primary tumors expressed low levels of ASZ1 possessed median PPS of 31.8 months, while patients whose tumors expressed high levels of ASZ1 possessed median PPS of 16.92 months. This difference in PPS based on ASZ1 tumor expression in patients with breast cancer was statistically significant (Figure 2, Chart 3; logrank $p$-value: 0.023; hazard ratio: $1.51(1.06-2.15))$.

Thus, by mining published microarray data ${ }^{4,5}$ in an unbiased and systematic fashion, we identified the ankyrin repeat, SAM and basic leucine zipper domain containing 1, encoded by ASZ1, as among the genes whose expression was most different, transcriptome-wide, in the brain metastases of patients with breast cancer, when compared to primary tumors of the breast and in the primary tumor cells of patients with triple negative breast cancer when compared to normal mammary gland ductal cells; we observed significantly decreased expression of ASZ1 in brain metastases as compared to primary tumors of the breast. Further, we found a significant correlation between ASZ1 expression and patient survival outcomes, as post-progression survival was significantly greater in patients whose primary tumors expressed lower levels of ASZ1 as compared to patients whose primary tumors expressed higher levels of ASZ1.

\section{Discussion}

We provided evidence here that the ankyrin repeat, SAM and basic leucine zipper domain containing 1, encoded by ASZ1, is among the genes whose expression is most different in the brain 


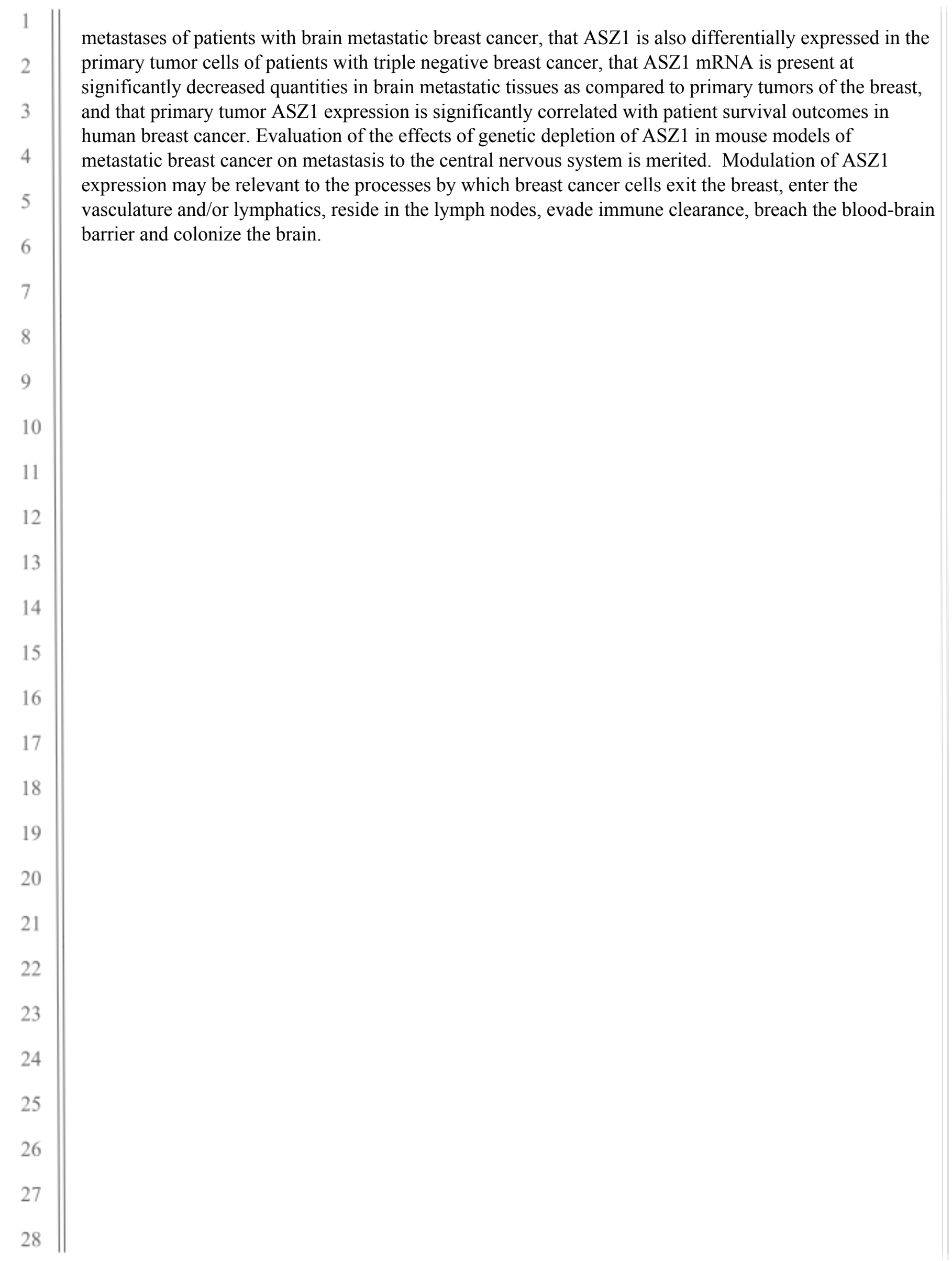




\section{References}

1. Lin, N.U., Amiri-Kordestani, L., Palmieri, D., Liewehr, D.J. and Steeg, P.S., 2013. CNS metastases in breast cancer: old challenge, new frontiers.

2. Bendell, J.C., Domchek, S.M., Burstein, H.J., Harris, L., Younger, J., Kuter, I., Bunnell, C., Rue, M., Gelman, R. and Winer, E., 2003. Central nervous system metastases in women who receive trastuzumab-based therapy for metastatic breast carcinoma. Cancer, 97(12), pp.2972-2977.

3. Tsukada, Y., Fouad, A., Pickren, J.W. and Lane, W.W., 1983. Central nervous system metastasis from breast carcinoma autopsy study. Cancer, 52(12), pp.2349-2354.

4. Weigman, V.J., Chao, H.H., Shabalin, A.A., He, X., Parker, J.S., Nordgard, S.H., Grushko, T., Huo, D., Nwachukwu, C., Nobel, A. and Kristensen, V.N., 2012. Basal-like Breast cancer DNA copy number cancer research and treatment, 133(3), pp.865-880.

5. Komatsu, M., Yoshimaru, T., Matsuo, T., Kiyotani, K., Miyoshi, Y., Tanahashi, T., Rokutan, K., Yamaguchi, R., Saito, A., Imoto, S. and Miyano, S., 2013. Molecular features of triple negative breast cancer cells by genome-wide gene expression profiling analysis. International journal of oncology, 42(2), pp.478-506.

6. Awada, A., Colomer, R., Inoue, K., Bondarenko, I., Badwe, R.A., Demetriou, G., Lee, S.C., Mehta, A.O., Kim, S.B., Bachelot, T. and Goswami, C., 2016. Neratinib plus paclitaxel vs trastuzumab plus paclitaxel in previously untreated metastatic ERBB2-positive breast cancer: the NEfERT-T randomized clinical trial. JAMA oncology, 2(12), pp.1557-1564.

7. Györffy, B., Lanczky, A., Eklund, A.C., Denkert, C., Budczies, J., Li, Q. and Szallasi, Z., 2010. An online survival analysis tool to rapidly assess the effect of 22,277 genes on breast cancer prognosis using microarray data of 1,809 patients. Breast cancer research and treatment, 123(3), pp.725-731. 


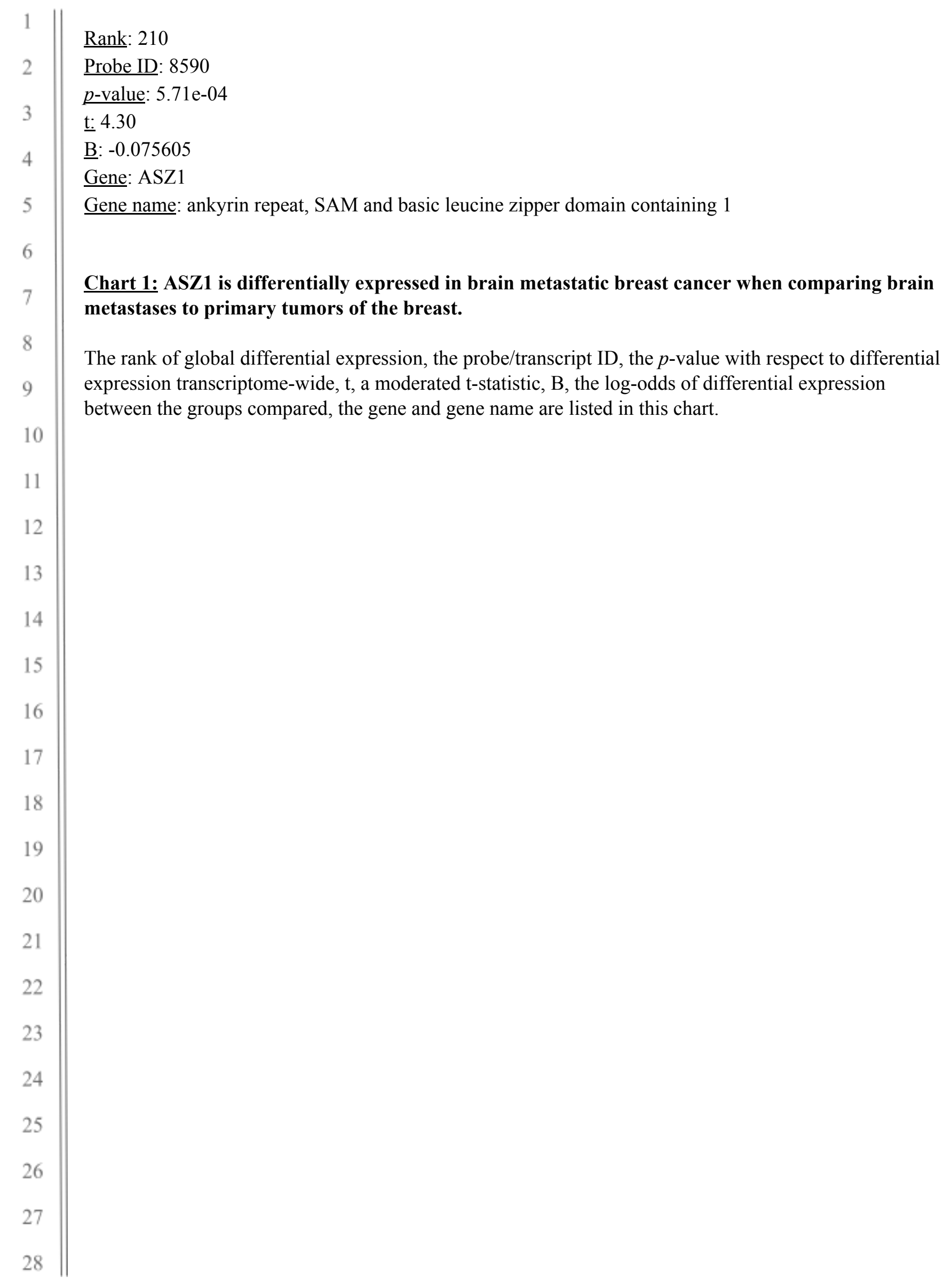




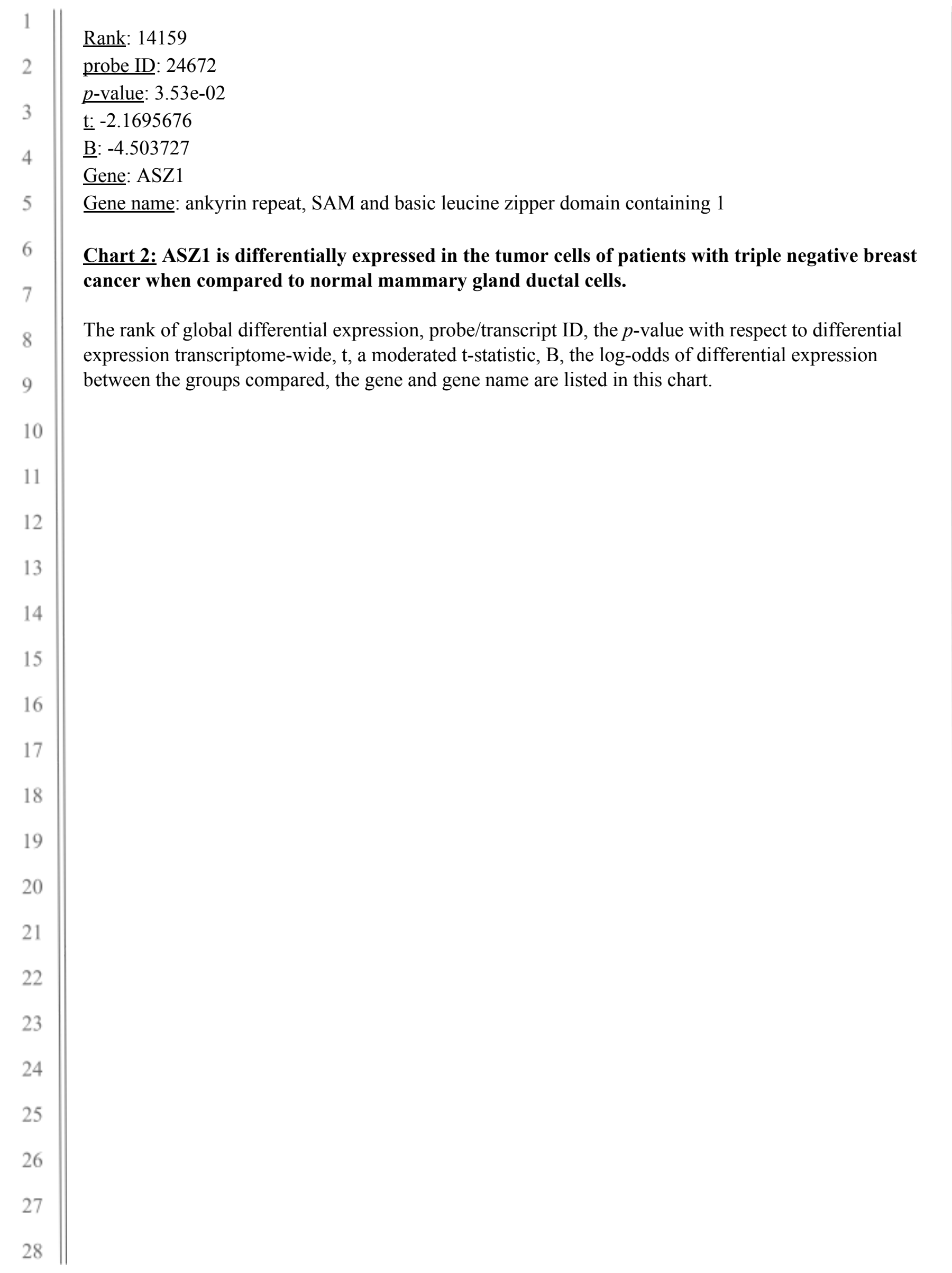




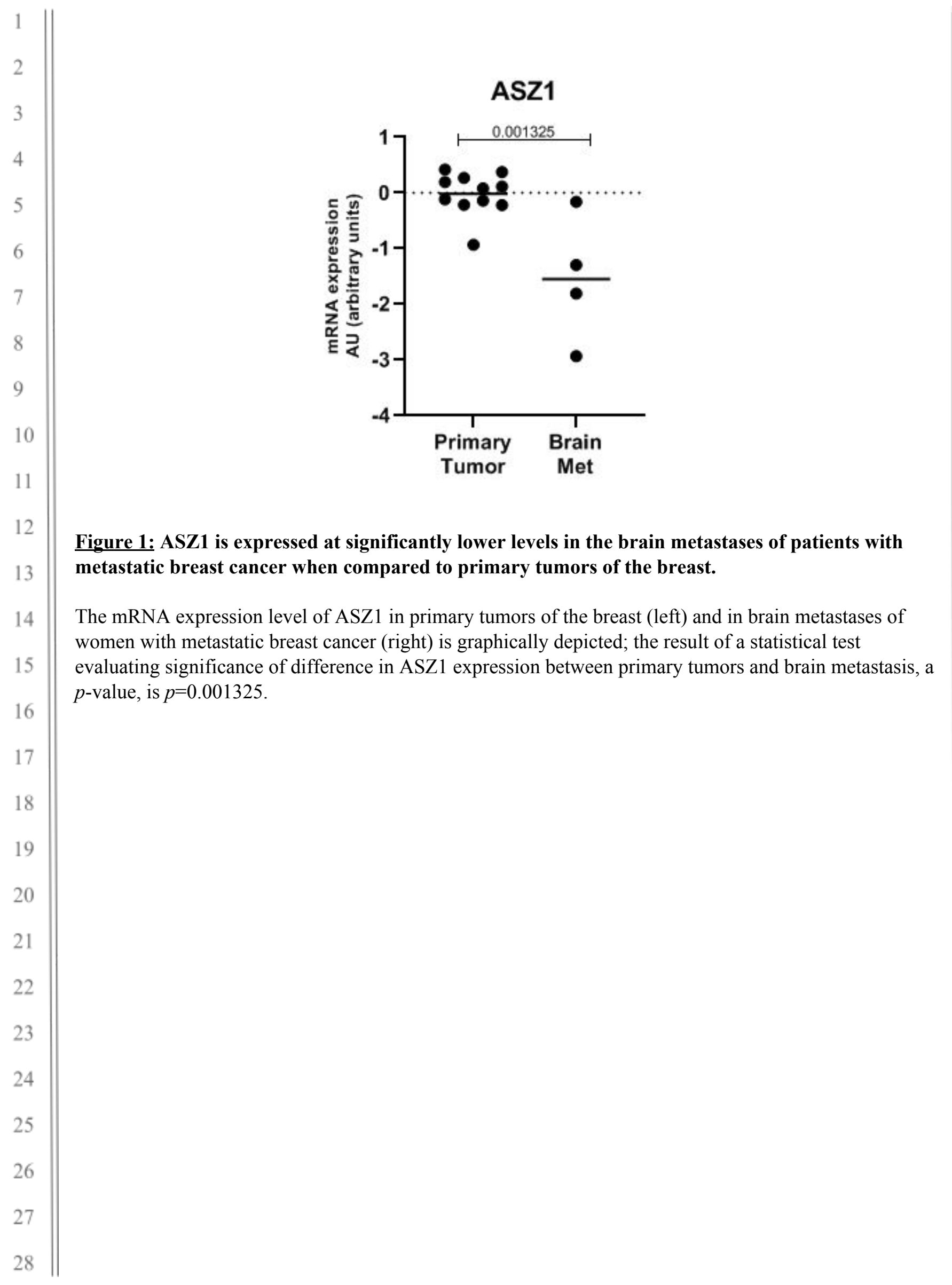




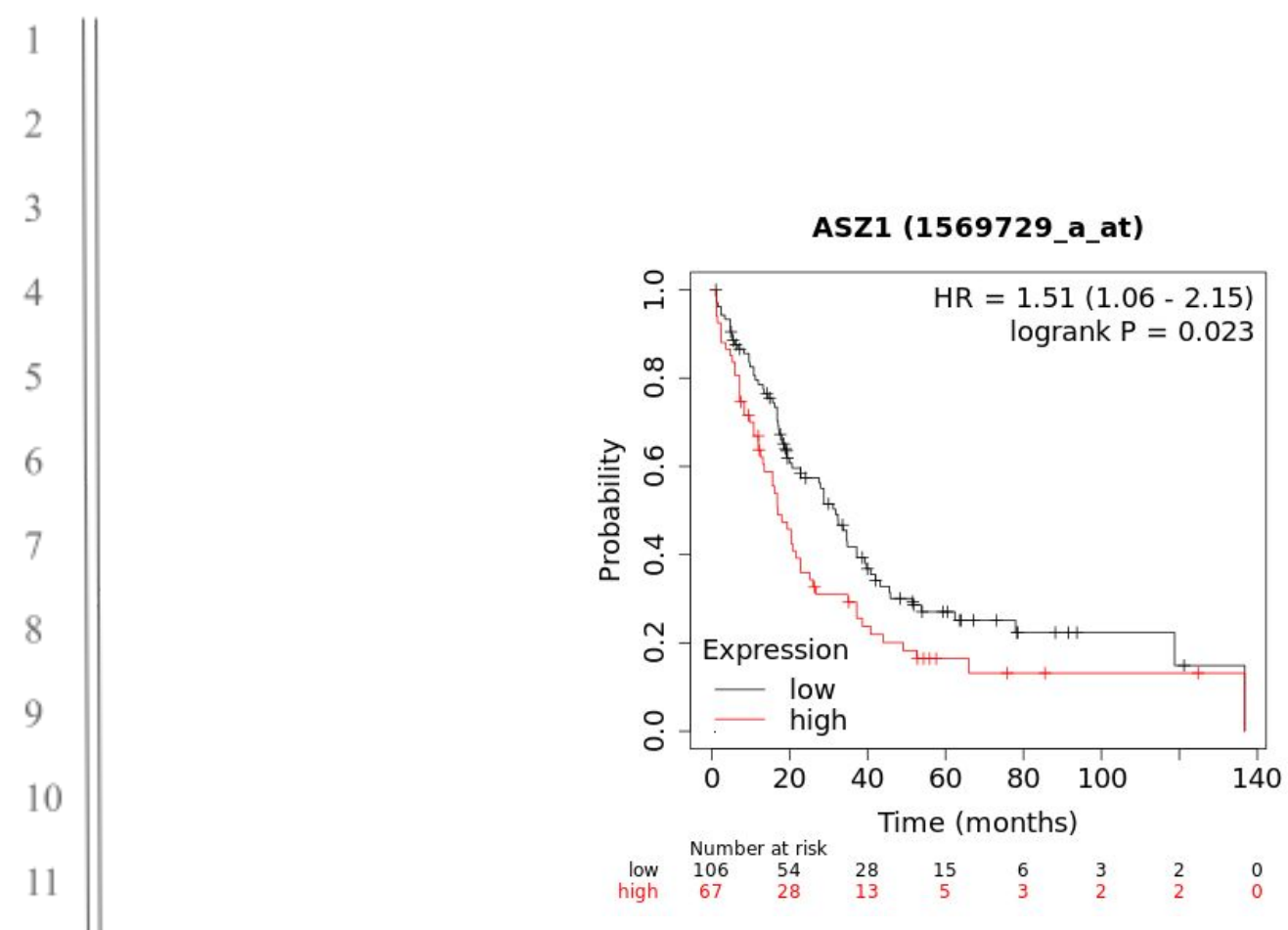

Figure 2: Significant correlation between ASZ1 primary tumor expression and post-progression survival in patients with breast cancer.

Depicted in this Kaplan-Meier plot is the probability of post-progression survival for $n=173$ total patients stratified into two groups, based on low or high expression of ASZ1 in patient primary tumors. The log rank $p$-value denoting statistical significance of difference in post-progression survival when comparing the two groups, as well as hazard ratio for this comparison is listed above. Listed below is the number of patients at risk (number of patients alive) per interval, after stratification based on ASZ1 expression; in the first interval, number at risk is number of patients alive; in each subsequent interval, number at risk is the number at risk less those who have expired or are censored. 


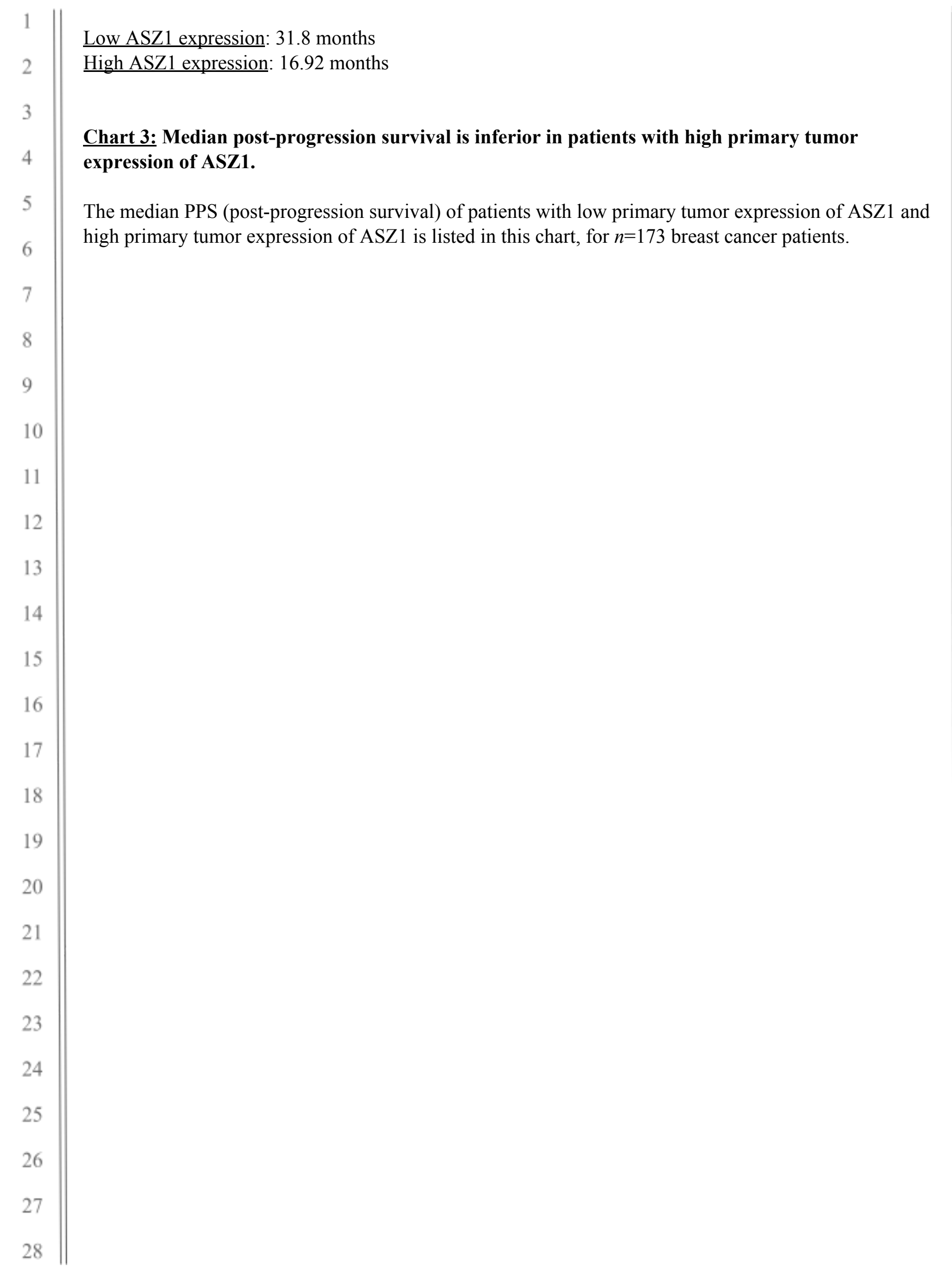

